\title{
A cucumber green mottle mosaic virus vector for virus-induced gene silencing in cucurbit plants
}

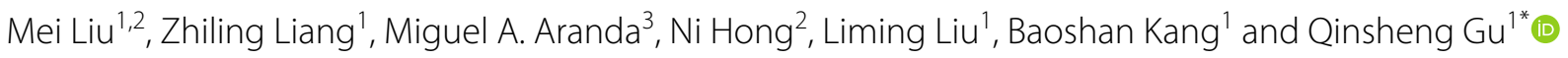

\begin{abstract}
Background: Cucurbits produce fruits or vegetables that have great dietary importance and economic significance worldwide. The published genomes of at least 11 cucurbit species are boosting gene mining and novel breeding strategies, however genetic transformation in cucurbits is impractical as a tool for gene function validation due to low transformation efficiency. Virus-induced gene silencing (VIGS) is a potential alternative tool. So far, very few ideal VIGS vectors are available for cucurbits.

Results: Here, we describe a new VIGS vector derived from cucumber green mottle mosaic virus (CGMMV), a monopartite virus that infects cucurbits naturally. We show that the CGMMV vector is competent to induce efficient silencing of the phytoene desaturase (PDS) gene in the model plant Nicotiana benthamiana and in cucurbits, including watermelon, melon, cucumber and bottle gourd. Infection with the CGMMV vector harboring PDS sequences of 69-300 bp in length in the form of sense-oriented or hairpin cDNAs resulted in photobleaching phenotypes in N. benthamiana and cucurbits by PDS silencing. Additional results reflect that silencing of the PDS gene could persist for over two months and the silencing effect of CGMMV-based vectors could be passaged.
\end{abstract}

Conclusions: These results demonstrate that CGMMV vector could serve as a powerful and easy-to-use tool for characterizing gene function, controlling viral pathogens or even performing resistance breeding in cucurbits. Moreover, this study will possess considerable important reference value for developing different viral vectors.

Keyword: Cucumber green mottle mosaic virus, Viral vector, Virus-induced gene silencing, Cucurbit plants

\section{Background}

The family Cucurbitaceae is second only after the Solanaceae for its economic importance among horticultural species worldwide, containing about 1000 species in 96 genera [1]. Cucurbits are generally prized for their delicious fruits, which might be low in nutritional value, but can be significant dietary sources of minerals and vitamins, some even with medical values. Watermelon (Citrullus lanatus), melon (Cucumis melo), cucumber

\footnotetext{
*Correspondence: guqinsheng@caas.cn

${ }^{1}$ Zhengzhou Fruit Research Institute, Chinese Academy of Agricultural

Sciences, Zhengzhou 450009, People's Republic of China

Full list of author information is available at the end of the article
}

(Cucumis sativus) and bottle gourd (Lagenaria siceraria) all belong to the family Cucurbitaceae with a significant impact on human nutrition [2].

With the increase of consumer's demand for high-quality fruits and vegetables and the improvement of agricultural production, it is urgent to explore genes encoding important agronomic traits in crop species, in order to breed elite, disease-resistant and featured varieties. So far, 11 reference genomes of cucurbit species [3] including watermelon [4], melon [5] and cucumber [6] have been published, which have boosted gene mining and gene function research. However, the genetic transformation of cucurbit plants is time-consuming and laborintensive, with extremely low efficiencies [7]. As a tool

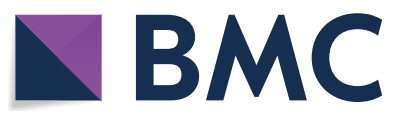

(c) The Author(s) 2020. This article is licensed under a Creative Commons Attribution 4.0 International License, which permits use, sharing, adaptation, distribution and reproduction in any medium or format, as long as you give appropriate credit to the original author(s) and the source, provide a link to the Creative Commons licence, and indicate if changes were made. The images or other third party material in this article are included in the article's Creative Commons licence, unless indicated otherwise in a credit line to the material. If material is not included in the article's Creative Commons licence and your intended use is not permitted by statutory regulation or exceeds the permitted use, you will need to obtain permission directly from the copyright holder. To view a copy of this licence, visit http://creativeco mmons.org/licenses/by/4.0/. The Creative Commons Public Domain Dedication waiver (http://creativecommons.org/publicdomain/ zero/1.0/) applies to the data made available in this article, unless otherwise stated in a credit line to the data. 
for rapid gene function validation, virus-induced gene silencing (VIGS) is a good alternative to gene transformation because of its simplicity, high efficiency, and high throughput.

Gene silencing comprises transcriptional gene silencing (TGS) and post-transcriptional gene silencing (PTGS). VIGS, a type of PTGS, is a natural defense reaction that exists in a broad range of organisms. It confers resistance to foreign nucleic acid invasion through PTGS at the RNA level. Because it can silence a specific gene, leading to the loss of function of this gene, the potential of VIGS as a tool to analyze gene function has been quickly recognized [8].

In the past decades, a large number of viral vectors had been developed as powerful tools for the functional verification of genes in plants [9-16]. To date, three different RNA viruses have been developed as vectors for VIGS in cucurbit species, including apple latent spherical virus (ALSV) [11], tobacco ringspot virus (TRSV) [17] and tobacco rattle virus (TRV) $[18,19]$. However, very few applications of these vectors have been reported, implying that they have not been widely adopted for cucurbit gene function analyses. This might be related to their limited host range among cucurbits, cumbersome inoculation approaches and/or short silencing periods associated with insert instability. As a result, it is urgent to develop a vector with a wider range of cucurbit hosts, ease of inoculation, high silencing efficiency and longlasting gene silencing in cucurbit plants.

Cucumber green mottle mosaic virus (CGMMV) is an important pathogen infecting cucurbit plants in natural conditions [20]. We have successfully constructed a full-length infectious clone of CGMMV, which can systemically infect plants of various cucurbit species such as watermelon, melon, cucumber and bottle gourd [21], making it a good candidate for VIGS vector development in cucurbits. CGMMV is a member of the genus Tobamovirus, and has a positive single-stranded genomic RNA of approximately $6.4 \mathrm{~kb}$ [22]. The CGMMV genome possesses four open reading frames (ORFs) encoding two replication-related proteins, one movement protein (MP), and one coat protein (CP). Only the $129 \mathrm{KDa}$ and $186 \mathrm{KDa}$ of replication-related proteins are translated directly from the genomic RNA, whereas the $29-\mathrm{KDa}$ $\mathrm{MP}$ and the 17.4-KDa CP are translated from two subgenomic RNAs. There is an overlap between the MP and CP ORFs [22]. Viral vectors based on CGMMV for expressing foreign genes have been constructed. Multiple cloning sites (MCS) were inserted adjacent to the $\mathrm{CP}$ ORF, and the CP stop codon was altered to express the hepatitis B surface antigen and a Dengue virus Epitope so that 20 and 44 foreign amino acids, respectively, were expressed [23, 24]. Tobacco mosaic virus (TMV), another member of the genus Tobamovirus, has been widely studied as a model in this genus. TMV has successfully been developed as a VIGS vector by including an additional duplicated copy of the CP subgenomic promoter (SGP) in the viral genome [25]. The CGMMV genome is similar to that of TMV, and thus it was thought that methods similar to those used for TMV could be used to create vectors based on CGMMV; unfortunately, results in this regard varied largely [26, 27], therefore the strategy of SGP duplication and information on the subgenomic promoter have not been fully exploited for constructing CGMMV-based viral vectors.

CGMMV has not been reported for its development for VIGS, although it has been exploited as a transient gene expression vector by read through translation or adding an additional subgenomic $\mathrm{CP}$ promoter. In this study, we developed a new CGMMV-based VIGS vector, which produces very mild viral symptoms and efficiently triggers gene silencing in the model plant $N$. benthamiana and cucurbit plants such as watermelon, melon, cucumber and bottle gourd.

\section{Results}

\section{Construction of a set of CGMMV vectors}

The first step in constructing a VIGS vector is to determine the insertion site of a foreign gene fragment. For CGMMV, we can place the insertion site behind the viral MP gene or between the CP gene stop codon and the $3^{\prime}$ non-coding region. For the set of vectors built and tested in this study, we chose the latter as the first strategy. We used HindIII restriction sites at the $3^{\prime}$ end of the CP as the insertion site for constructing our first VIGS vectorpV1a23, a pXT1-CGMMV derivative missing the first restriction HindIII site at $5^{\prime}$ terminus and with the 159th amino acid of the CP mutated to a stop codon (Fig. 1a). Cucurbit plants inoculated with this vector showed viral symptoms on upper leaves similar to those of plants inoculated with the pXT1-CGMMV, and a progeny of CGMMV could be detected by DAS-ELISA and RT-PCR in these leaves (Fig. 1b, c; Additional file 1: Table S2).

Secondly, we determined the impact of a duplicated copy of the CP SGP in the viral genome on infections of VIGS [25], therefore we build vectors pV61, pV92 and pV112 (Fig. 2a). Viral symptoms could be observed on upper leaves of plants inoculated with vectors $\mathrm{pV} 92$ and pV112, whereas plants inoculated with pV61 did not develop viral symptoms and CGMMV could not be detected by DAS-ELISA. These results revealed that vectors pV92 and pV112 could infect plants systemically while pV61 could not.

Furthermore, our previous work revealed that the CP RNA transcription level was significantly enhanced when 105 nucleotides were retained before the $\mathrm{CP}$ 
a pV1a23

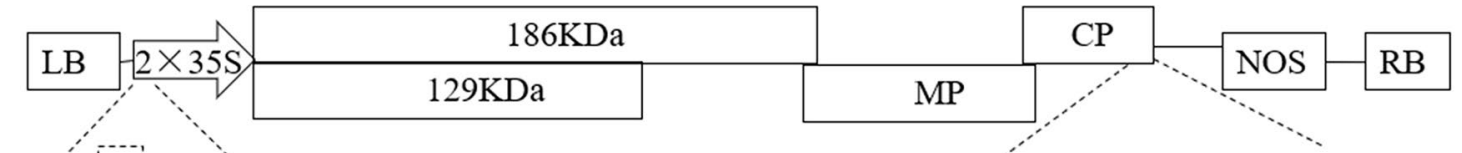

AAGCTTGCATGCC

HindIII $\underset{\text { Delete:TT }}{\longrightarrow}$ AAGC

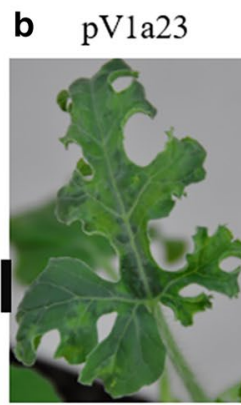

pV1a23-PDS114 pXT1-CGMMV
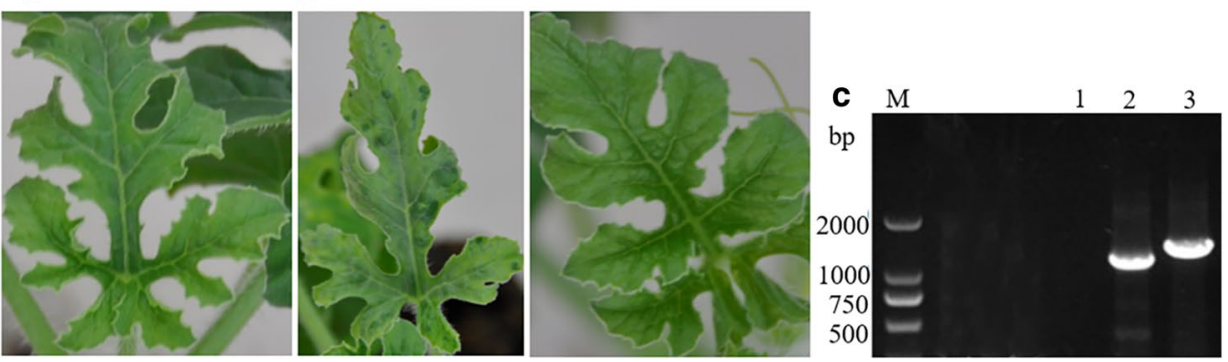

Fig. 1 Engineering of CGMMV as a VIGS vector with an insertion site behind the CP. a Schematic representation of the pV1a23 vector with a restriction enzyme site (HindIII) for insertion of gene fragments. b Viral symptoms on upper non-inoculated leaves caused by pV1a23 similar to those of plants inoculated with the pXT1-CGMMV. Photobleaching was absent on plants inoculated with pV1a23-PDS114. Bar =1 cm. c RT-PCR detection of viral RNA from pV1a23 and pV1a23-PDS114 in watermelon. M, Marker2000; CK, negative control; 1, 2 and 3 indicate healthy control and plants inoculated with pV1a23 and pV1a23-PDS114, respectively

transcription starting site (TSS) and that the sequence from the 71st base to the 91st base upstream of the CP TSS plays a key role in CP SGP activity [28]. Based on these results, we built pV190, a pXT1-CGMMV derivative that contains a direct repeat of the 190-bp putative CGMMV CP SGP and a single restriction site (BamHI) between the duplicated CP SGPs (Fig. 2a). N. benthamiana and cucurbit plants inoculated with pV190 developed very mild symptoms on upper leaves, which were clearly milder than those of plants inoculated with the pXT1-CGMMV (Fig. 2b). However, the pV190 genomic RNA could be detected by RT-PCR (Fig. 2c), indicating that it could replicate and move systemically.

\section{Silencing effects of PDS fragments inserted in the sense orientation or conforming a hairpin}

To determine whether pV1a23, pV92, pV112 and pV190 can be used to induce gene silencing in cucurbits, we chose to target $P D S$ because it can result in striking photo-bleaching when silenced. Cucurbit $P D S$ fragments with various lengths $(114,150,213$ and $300 \mathrm{bp})$ were amplified by selecting the region with the highest conservation. The sequence similarity of the 114-, 150- and 213-bp fragments in the four cucurbit species was approximately 97\% (Additional file 2: Fig. S1a-c), While that of 300-bp fragments was $98.4 \%$, but the fragment from watermelon contains an insertion of $30 \mathrm{bp}$ (Additional file 2: Fig.S1d).
The PDS fragments of 114-bp, 213-bp and 300-bp were inserted in the sense orientation at the HindIII cloning site of pV1a23 to produce pV1a23-PDS114, pV1a23PDS213 and pV1a23-PDS300, respectively. Similarly, the $P D S$ fragments of $150 \mathrm{bp}$ and $213 \mathrm{bp}$ were also inserted in the sense orientation at the $\mathrm{BamHI}$ cloning site of pV92 and pV112 to produce pV92-PDS150, pV92-PDS213 and pV112-PDS150 and pV112-PDS213, respectively. The PDS fragments of $150 \mathrm{bp}, 213 \mathrm{bp}$ and $300 \mathrm{bp}$ were inserted in the sense orientation at the $\mathrm{BamHI}$ cloning site of pV190 to produce pV190-PDS150, pV190-PDS213 and pV190-PDS300, respectively.

To verify the silencing efficiency of these vectors, cucurbit plants (watermelon, melon, cucumber and bottle gourd) were subjected to Agrobacterium-mediated inoculation. Results revealed that pV1a23-PDS300 lost the ability of systemic and local infection, pV1a23PDS213 was only able to infect locally and pV1a23PDS114 could produce systemic and local infection (Additional file 1: Table S2), but failed inducing photobleaching (Fig. 1b), although the 114-bp PDS gene fragment was stable (Fig. 1c). Therefore, pV1a23 can not be used for VIGS. Photobleaching could be observed in the inoculated plants with pV92-PDS150 and pV112-PDS150 (Additional file 1: Table S2). However, pV92-PDS213and pV112-PDS213-infected plants did not display any photobleached phenotype and the presence of CGMMV 


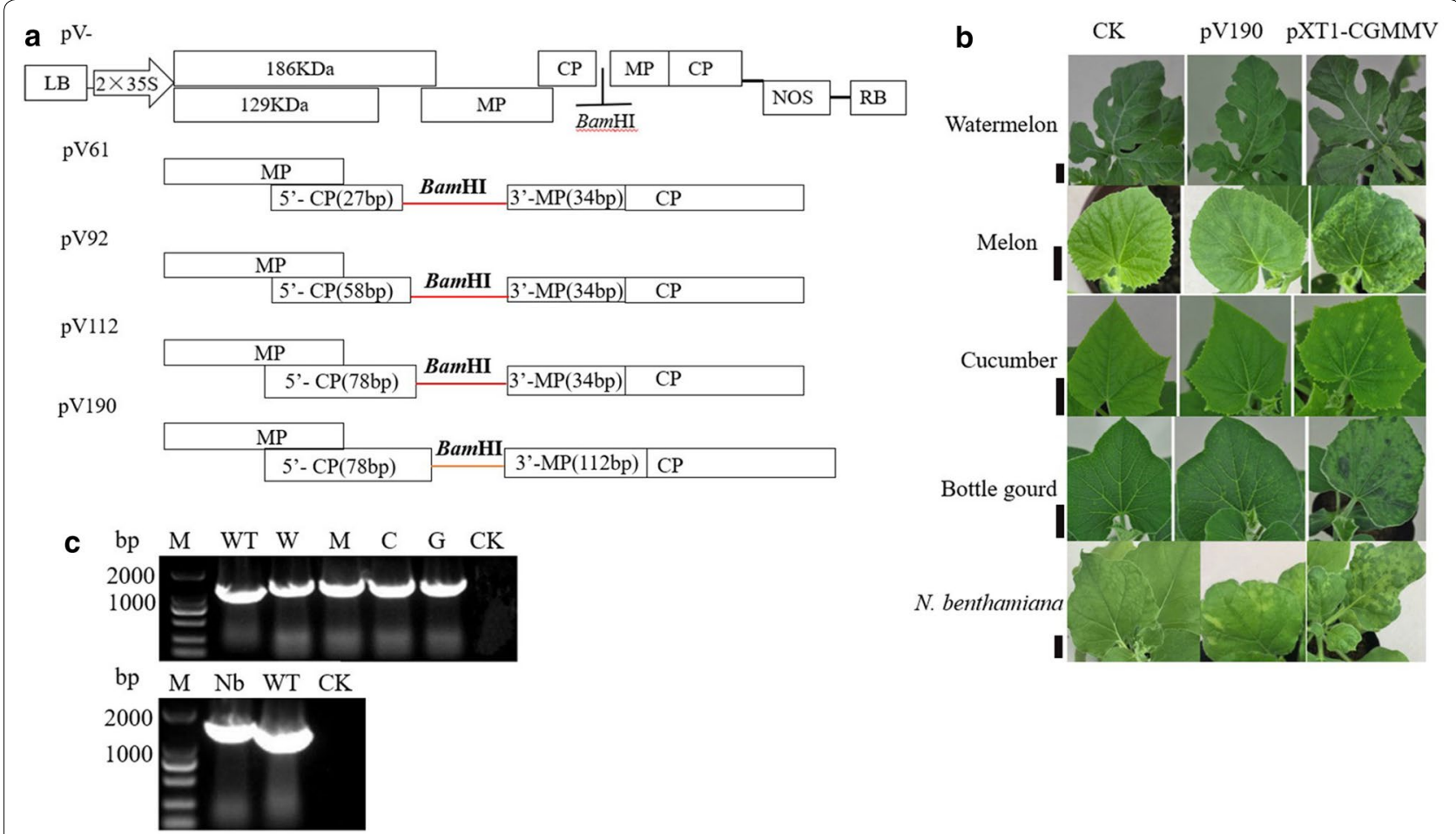

Fig. 2 Engineering of CGMMV as a VIGS vector with different size CP subgenomic promoters. a Schematic representation of pV61, pV92, pV112 and pV190. pV- is a PXT1-CGMMV derivative that contains a direct repeat of the 61-, 92-, 112- and 190-bp putative CGMMV CP subgenomic promoter and a restriction enzyme site (BamHI) between CP subgenomic promoters. b pV190 caused mild systemic symptoms on cucurbits and $N$. benthamiana. Bar $=1,3,3,3$ and $1 \mathrm{~cm}$, respectively. $\mathbf{c}$ RT-PCR detection of viral RNA showing that PXT1-CGMMV and pV190 are infectious in cucurbits and N. benthamiana. M, Marker2000; WT, wild type (pXT1-CGMMV); CK, negative control; W, M, C, G and Nb indicate watermelon, melon, cucumber, bottle gourd and N. benthamiana, respectively

in the upper leaves was not observed (Additional file 1: Table S2). The cotyledons of watermelon, melon, cucumber and bottle gourd seedlings were inoculated with pV190-PDS150, pV190-PDS213 and pV190-PDS300. Photobleaching was first observed among all plants inoculated with different vectors on the 4th true leaves (L4) in watermelon at about $19 \mathrm{dpi}$, on the 3rd leaves (L3) in melon and bottle gourd at $12 \mathrm{dpi}$ (Fig. 3a), and on the 5 th true leaves of cucumber at $28 \mathrm{dpi}$. Further, photobleaching was observed up to 32, 20 and 39 dpi in watermelon, melon and cucumber plants, respectively (Fig. 3b). About $70 \%$ of the inoculated plants showed a photobleaching phenotype. Total RNA was extracted from leaves of the plants inoculated with different vectors displaying the most obvious photobleaching (Fig. 4a) and the accumulation of PDS transcripts was quantified by qRT-PCR. The results showed that the expression levels of PDS had no significant differences between pV190-infected (Empty Vector, EV) and noninfected (NI) leaves, demonstrating that pV190 did not significantly affect PDS expression (Fig. 4b). The PDS mRNA transcript levels in photobleached leaves was reduced by approximately $79 \%$,
$81 \%$ and $89 \%$ in watermelon, $78 \%, 76 \%$ and $81 \%$ in melon, $83 \%, 87 \%$ and $89 \%$ in bottle gourd, and $82 \%, 64 \%$ and $88 \%$ in cucumber infected with pV190-PDS150, pV190PDS213 and pV190-PDS300, respectively, compared to plants infected with pV190 (Fig. 4b).

Further, to improve silencing efficiency, we inserted a PDS fragment forming a hairpin structure of 69-bp into pV190 to produce the pV190-PDS69 vector. We first observed photobleaching phenotype among all plants inoculated with different vectors on the L5 in watermelon at $17 \mathrm{dpi}$ and photographed at $19 \mathrm{dpi}$, on the L2 in melon at $10 \mathrm{dpi}$ and at $12 \mathrm{dpi}$, on the L3 in bottle gourd at $11 \mathrm{dpi}$ and at $22 \mathrm{dpi}$ (Fig. 3). Photobleaching was first observed one or two days earlier in plants infected with pV190-PDS69 than in plants infected with any other vector. The PDS mRNA levels declined 76\%, 41\%, 42\% and $83 \%$ in watermelon, melon, cucumber and bottle gourd, respectively (Fig. 4b).

\section{Stability of the 69-300-bp PDS fragments in pV190}

We observed that the silencing phenotype of the PDS gene could persist for over 2 months in bottle gourd 


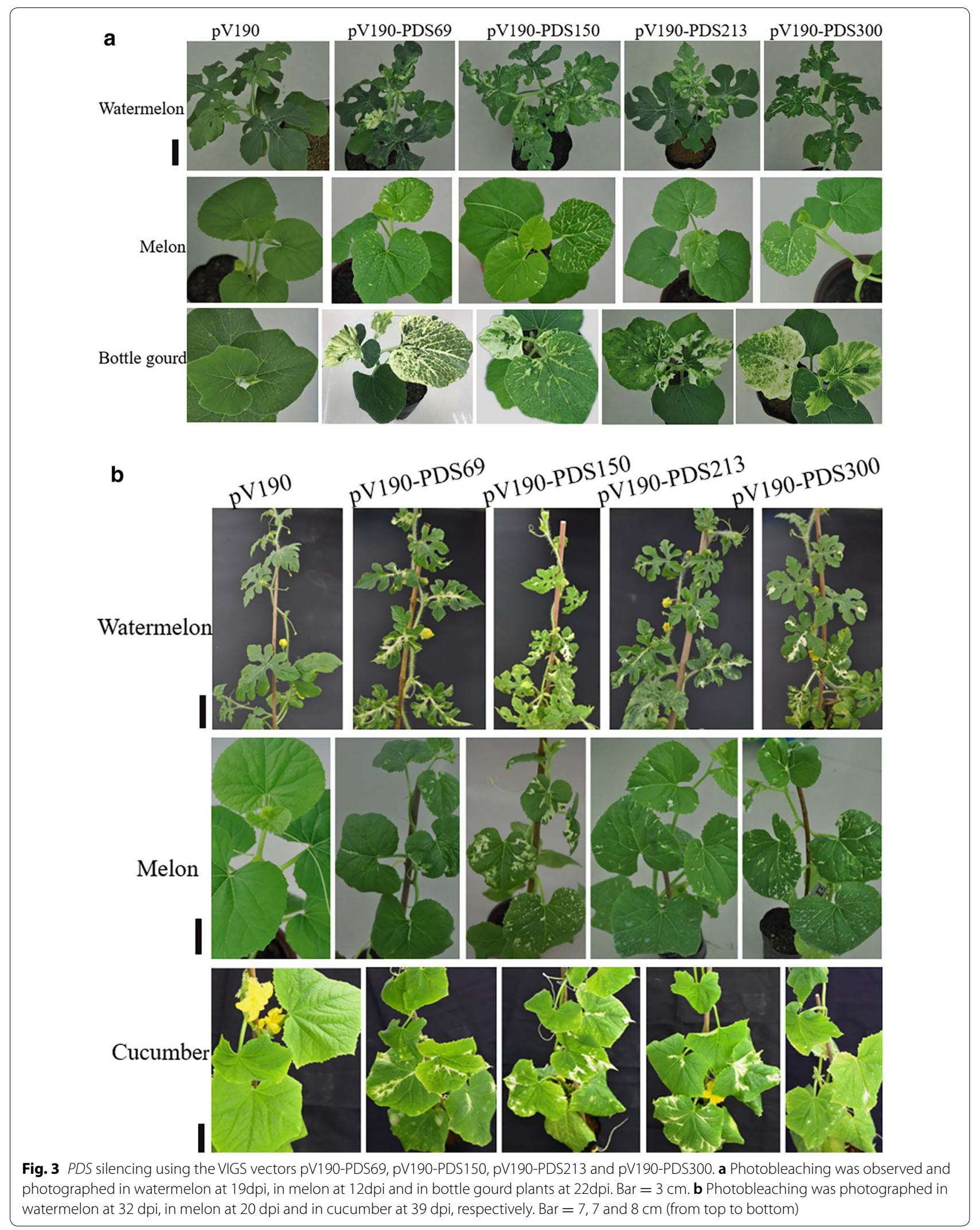




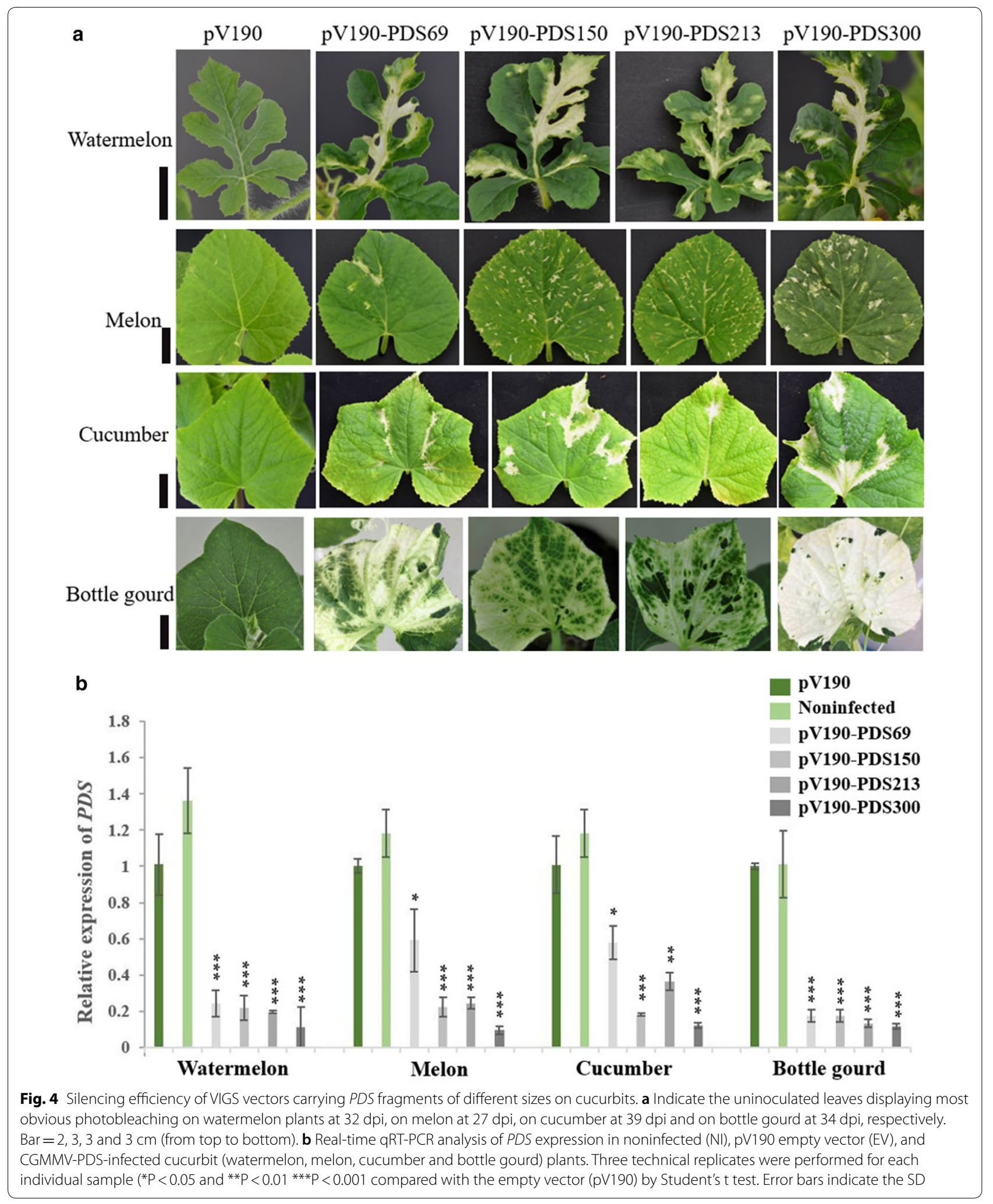


(Fig. 5a). Photobleaching was not uniform from bottom to top of bottle gourd leaves (Fig. 5b). To evaluate the stability of the PDS fragment in CGMMV-vectors, RTPCR was performed on total RNA extracted from bottle gourd leaves L6, L7 and L9 for pV190-PDS69, L4 and L11 for pV190-PDS300, L4 and L12 for pV190-PDS213, and L7, L9 and L10 for pV190-PDS300 (Fig. 5b). The result showed the 150-bp and 213-bp PDS fragments were stable across all analyzed leaves. The 69-bp dsRNA hairpin structure could not be detected across all leaves, whereas L9 and L10 samples from pV190-PDS300-infected bottle gourd contained deletions of the 300-bp PDS fragment to different extents (the deletion in L9 was less than the L10) (Fig. 5c). The relative expression of the PDS gene in the above same leaves was measured by qRT-PCR. RTPCR results corresponded well with the PDS relative expression level measured by qRT-PCR, with less silencing observed as the extent of deletions increased (Fig. 5c, d). For instance, pV190-PDS69 caused PDS transcripts to be reduced by $83 \%, 80 \%$ and $65 \%$ in the L6, L7, L9, respectively, and pV190-PDS300 caused PDS transcripts to be reduced by $87 \%, 81 \% 73 \%$ and $65 \%$ in the $L 7, L 8$, L9 and L10, respectively (Fig. 5d). Results of stability of the 69-300-bp PDS fragments in pV190 in watermelon, melon and cucumber were consistent with those in bottle gourd. The expression of PDS in the youngest analyzed leaves was still down-regulated (Fig. 5d), indicating that these vectors have sufficient stability to be used to characterize gene functions in cucurbit plants.

\section{The silencing effect of CGMMV-based vectors could be passaged}

To verify whether the silencing vectors can be passaged, the sap of leaves with obvious photobleaching was used to rub-inoculate cotyledons and the L1 of melon plants. Photobleaching occurred on uninoculated leaves as early as $9 \mathrm{dpi}$ and was photographed at $14 \mathrm{dpi}$ (Fig. 6a). PDS expression levels were tested on L5 of passaged plants. We observed that PDS relative expression was reduced by $32 \%, 52 \%, 25 \%$ and $85 \%$ in pV190-PDS69, -PDS150, -PDS213 and -PDS300, respectively (Fig. 6b), confirming that the silencing effect of CGMMV-based vectors could be passaged.

\section{CGMMV-based VIGS in N. benthamiana}

Nicotiana benthamiana is an important experimental host for CGMMV. We utilized two different lengths of $P D S$ fragments which were amplified by selecting conserved regions of $P D S$ gene sequences in $N$. benthamiana to test whether CGMMV is competent to induce gene silencing in $N$. benthamiana plants. At $14 \mathrm{dpi}$, a little weak photobleaching could be observed in the upper leaves of all plants inoculated with either pV190NbPDS146 or pV190-NbPDS215 (Additional file 3: Fig. S2a). Consistently, qRT-PCR results showed that the expression of PDS in pV190-NbPDS146- and pV190NbPDS215-infected leaves was reduced by $60 \%$ and $34 \%$, respectively, compared with the pV190 infected leaves (Additional file 3: Fig. S2b).

\section{Discussion}

In this study, we successfully developed a new CGMMVbased VIGS vector which could be used to silence endogenous genes in cucurbit plants. Using this viral system, we successfully silenced $P D S$ in cucurbits including watermelon, melon, cucumber and bottle gourd and in the model plant $N$. benthamiana. To our best knowledge, this is the first time that CGMMV has been engineered as a VIGS vector, although it has been exploited for protein overexpression.

During the process of modifying the CGMMV genome to produce a VIGS vector, we observed that the insertion sites of the gene fragment determined the viability, stability, insert size and silencing efficiency of the vector; our work showed that a duplicated copy of the 190-bp putative CGMMV CP SGP was essential for silencing. We first tried to place the foreign gene insertion site downstream of the viral CP gene. Results demonstrated that the insertion site between the CGMMV CP gene stop codon and the 3 ' non-coding region was not suitable for constructing the VIGS vector. TMV is a member of the genus Tobamovirus and has successfully been developed as a VIGS vector by utilizing the strategy of subgenomic expression [25]. Because CGMMV is also a member of the genus Tobamovirus and CGMMV infectious clone containing the green fluorescence protein (GFP) reporter gene has been successfully constructed, the GFP gene was located in between MP and CP [27]. Thus, we adopted a similar strategy as reported for TMV to generate a CGMMV VIGS vector and explored different lengths of the duplicated region. For the impact of length of 61-, 92-, 112- and 190-bp duplicated copy of the putative CGMMV CP SGP on silencing, only when the modified CGMMV-based vector contained a 190-bp duplicated copy of the putative CGMMV CP SGP, the $P D S$ gene fragments could induce a robust silencing phenotype. These results suggest that it is necessary to create an additional fully competent subgenomic promoter to drive the transcription of the VIGS target sequence and for providing the vector with the ability to systemically infect plants [29].

Vectors containing duplicated sequences frequently suffer partial or complete loss of inserted sequences, particularly when the insert size is large [30, 31]. We tested the effect of the length and structure of inserts 


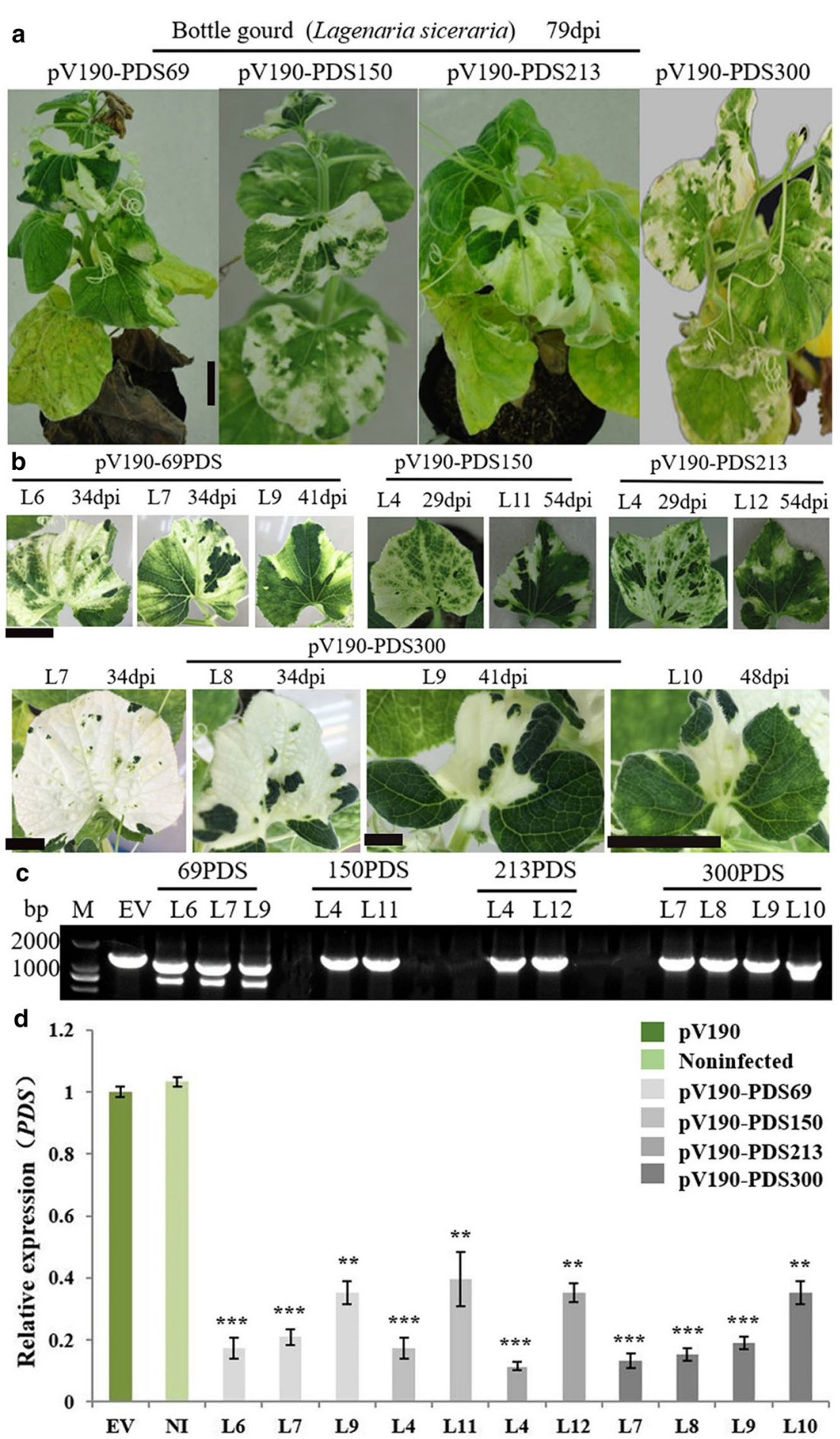

Fig. 5 Silencing efficiency and stability of the pV190 VIGS vector with different length inserts in bottle gourd. Fragments of 69 bp (dsRNA hairpin structure), 150 bp, 213 bp, 300 bp were separately cloned into pV190. a Silencing PDS using pV190 on bottle gourd plants produced photobleaching that persisted for over 70 days. Bar $=4 \mathrm{~cm}$, $\mathbf{b}$ Photobleaching on newly emerging leaves of bottle gourd plants caused by PDS silencing was observed at 29, 34, 41, 48 and 54 dpi, respectively. Bar $=2,1,2$ and $2 \mathrm{~cm}$. c RT-PCR assay to detect the presence of pV190 carrying PDS fragments of different sizes in systemic leaves. Samples from the 4rd leaf above the inoculated ( $L 4)$ were collected at $29 \mathrm{dpi}$ L6, $L 7$ and $L 8$ samples were collected at 34 dpi, L9 sample was collected at 41 dpi, L10 at 48 dpi, L11 and L12 at 54 dpi. M: Marker2000; EV: Empty vector (pV190). d Relative expression level of PDS mRNA in the above indicated leaves determined by real-time qRT-PCR 


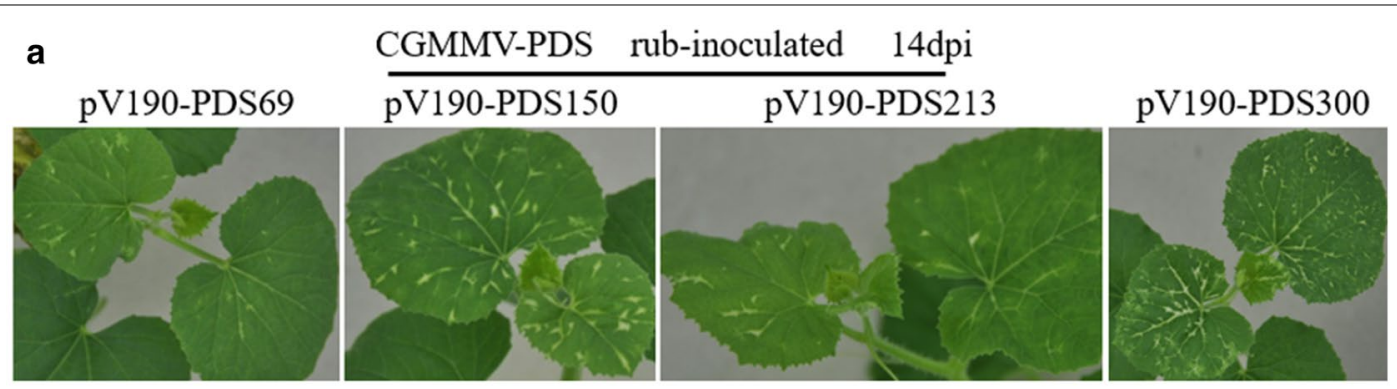

b

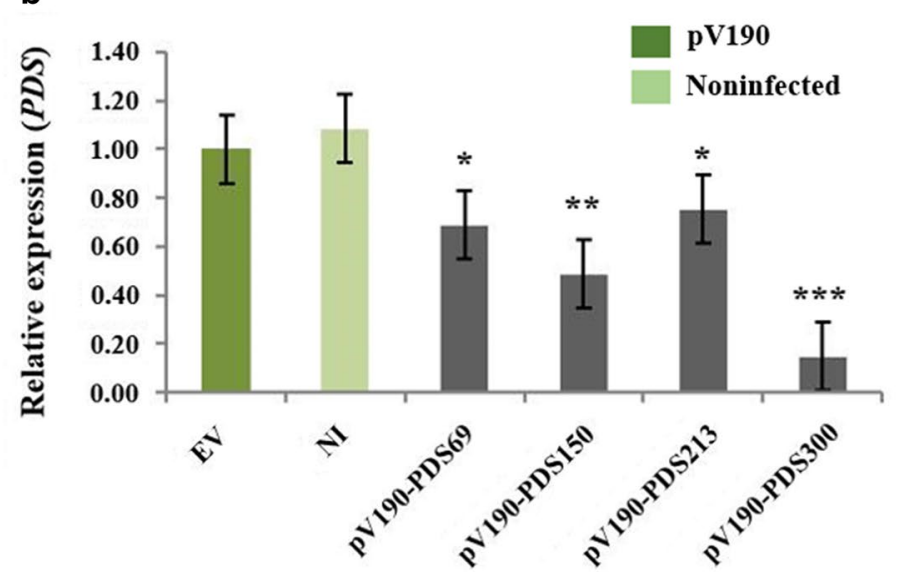

Fig. 6 The silencing effect of pV190-PDS69,-PDS150, -PDS213 and -PDS300 could be passaged. a Photobleaching caused by PDS silencing in systemic leaves of melon plants that were rub inoculated with sap from pV190-PDS69, -PDS150,-PDS213 and -PDS300-infected leaf tissue. The photobleaching phenotype was observed and photographed at 9dpi/14dpi. b Real-time qRT-PCR analysis of PDS expression in the 5 th leaf above the inoculated (L5) of noninfected (NI), pV190 empty vector (EV), and pV190-PDS69, -PDS150, -PDS213 and -PDS300-infected melon by mechanical inoculation

on silencing. Our results showed that the CGMMV vectors harboring the sense-oriented PDS gene sequence of 100-300 bp in length could effectively induce silencing in cucurbits, and efficiency was highest for the largest fragment, the 300-bp PDS gene fragment. It is worth mentioning the effect on silencing of the cDNA insert length in a tobacco rattle virus (TRV)-based vector [32]. The better silencing phenotype could be produced when the cDNA insert length was between 200 and $1300 \mathrm{bp}$, whereas inserts shorter than $190 \mathrm{bp}$ and longer than 1661 bp generated less siRNAs silencing less efficiently [32]. Not only the length of the insert affected silencing but also the structure of it has an impact on silencing. Expression of a hairpin-loop dsRNA structure could enhance the efficiency of VIGS [33]. This seems to be true for our CGMMV-based VIGS vector. The silencing efficiency of a 69-bp hairpin-loop structure was between that of the 150-bp and 300-bp sense constructs, but its silence phenotype appeared earlier. A direct 60-bp inverted-repeat sequence of the target gene that could fold as dsRNA strongly enhanced VIGS from foxtail mosaic virus (FoMV) [16]. However, in our work, a 60-bp inverted-repeat sequences of the PDS gene could not produce photobleaching, and CGMMV lost the ability of systemic infection (unpublished data). Therefore, these results suggest that the effect of length and structure of inserts on silencing varied with vectors from different viruses.

Furthermore, the stability of inserts in the pV190 vector were evaluated. The photobleaching phenotype was observed from the 3rd to the 11th leaves and PDS transcripts were reduced by about $80 \%$ and $20 \%$ in L4/L5 and L10/L11. About $70 \%$ of tested plants had a photobleaching phenotype which was stable and persisted for over 2 months. Stable photobleaching was also observed in plants mechanically inoculated with leaf sap prepared from L5/L6 of CGMMV-PDS inoculated plants. Here, it is worth mentioning that $P D S$ transcript abundance could be reduced by CGMMV-PDS vectors on the 3rd to 10th leaves of the tested plants. However, the photobleaching phenotype and the PDS transcript levels were not uniform in these leaves, and could produce a gradient from bottom to top. It has been reported that the phenomenon could be due to instability of the PDS gene fragment. 
RT-PCR analyses on all of these leaves showed that deletion of the PDS insert was hardly detected in samples with the sense inserts. Hence, we reasoned that genesilencing efficiency may be related to the accumulation of the foreign fragment-derived siRNAs [34, 35], but the specific mechanism of action remains unclear. In contrast, the full-length sequence of the insert in the pV190PDS69 vector could not be detected in photobleached leaves. This phenomenon may be explained by a systemic silencing signal that can be actively transmitted over long distances through the phloem to induce PDS gene silencing in young leaves [35-37], but the exact molecular form of a mobile RNA signal in the phloem still needs further research.

In addition, we tested whether pV190 can be used to induce gene silencing in $N$. benthamiana. Results showed that pV190 could infect $N$. benthamiana leaves, the uninoculated systemic leaves developed very mild symptoms. The pV190 vectors, harboring 146-bp and 215-bp PDS fragments could trigger silencing, but the photobleaching phenotype was not striking. The photobleaching phenotype also varied in cucurbit plants, with the most obvious phenotype in bottle gourd. We reasoned that the viral vector has different fitness for different hosts. The accumulation of siRNAs is a crucial factor for silencing efficiency, while the host species also contain crucial factors including the $D C L, R D R$ and $A G O$ genes [38-40].

Apple latent spherical virus was first described as a vector for gene silencing in cucurbits [11], and few additional studies have reported its application for VIGS in cucurbit plants. A new TRSV vector was recently reported [17]. More recently, the TRV-VIGS system has been used in cucumber and oriental melon $[18,19]$. Comparing ALSV, TRSV and TRV with CGMMV, the first three belong to multipartite virus families with a bipartite genome, while CGMMV is a monopartite virus [22], and therefore is easier to manipulate. The ALSV genome is expressed through polyprotein synthesis followed by proteolytic processing, which represents another layer of difficulty for high throughput functional genomics [41, 42]. A second major difference among the four cucurbit viral vectors is the inoculation method. A. tumefaciens infiltration is a simple, effective and convenient inoculation way [43, 44] and TRSV, TRV and CGMMV vectors are designed for agroinfiltration $[17,45]$. However, ALSV does not adopt this inoculation method. Host range is another major difference. TRSV cDNA clones are not infectious in watermelon or pumpkin [17]. Although TRV has been widely used as VIGS vector since it has a wide host range [46], its application for VIGS on cucurbits except cucumber and oriental melon have not been reported [18, 19]. Both ALSV and CGMMV vectors can be successfully used on common cucurbit plants such as watermelon, melon, cucumber and bottle gourd [11]. In short, the CGMMV-based VIGS vector pV190 is easy to handle during either the process of preparing a VIGS construct or for inoculations. Besides, it can systemically infect $N$. benthamiana and various cucurbit species and produce very mild viral symptoms in upper leaves of inoculated plants. Silencing phenotypes caused by pV190-based VIGS vectors were stable and could persist for at least one month.

\section{Conclusions}

CGMMV has a broad host range including 29 species, of which at least 16 belong to Cucurbitaceae [20]. Thus, the pV190 VIGS vector should have the potential for VIGS in many other plants although we only evaluated its application in $N$. benthamiana, watermelon, melon, cucumber and bottle gourd. Taken together, the CGMMV-based silencing system could be applied as a powerful biotechnological tool with a great potential for studying functional genomics in cucurbits. Future study will focus on obtaining insights into the molecular mechanism underlying the difference in silencing efficiency between different plants. In addition, the vector could serve as a basis to control devastating viral pathogens or carry out genetic engineering and molecular breeding.

\section{Materials and methods Plant materials}

The CGMMV experimental host $N$. benthamiana and cucurbits hosts (watermelon, melon, cucumber and bottle gourd) were used for VIGS of the PDS gene by CGMMV vectors in this study. Watermelon (Zhengkang 2), melon (Baimei), cucumber (Jinyan 4) and bottle gourd (Yongzhen1) seeds were obtained from Zhengzhou Fruit Research Institute (Zhengzhou, China), Xinjiang Academy of Agricultural Sciences (Xinjiang, China), Tianjin Academy of Agricultural Sciences (Tianjin, China) and Ningbo Academy of Agricultural Sciences (Ningbo, China), respectively. All cucurbit seeds were soaked in sterile water for $3-4 \mathrm{~h}$ at $50{ }^{\circ} \mathrm{C}$, then placed in Petri plates containing wetted filter cotton gauze at $28^{\circ} \mathrm{C}$ in darkness until seeds were germinated. Germinated seeds were planted into pots with nutrient matrix and grown in a growth chamber under $16 \mathrm{~h}$ light at $28^{\circ} \mathrm{C} / 8 \mathrm{~h}$ dark at approximately $22{ }^{\circ} \mathrm{C}$. The same conditions were used to grow inoculated plants (see below) with CGMMV vectors.

\section{Construction of the CGMMV-based vectors}

pV1a23 was constructed by site-directed mutagenic PCR using primer pairs DelHindIII-X/ S159Z-S and S159Z-X/DelHindIII-S (Additional file 4: Table S1). To construct pV61, pV92, pV112 and pV190 VIGS vectors, 
pXT1-CGMMV was modified by removing the CP start codon PCR using primer pairs CP-TC-F and CP-TC-R (Additional file 4: Table S1), resulting in the single-nucleotide substitution ATG to ACG. The resulting construct was named pXT1-CGACG. Further, DNA fragment 1 containing CGMMV nt 1-(5711-5840) (GenBank accession: KY753929) was amplified using pXT1-CGMMV as a template with primer pairs PXT1-F/(27B-34-R, 58B-34-R, 78B-34-R or 78B-99-R), whereas DNA fragment 2 containing CGMMV nt 5651/5716-6423 was amplified using pXT1-CGACG as a template with primer pairs PXT1-R /(27B-34-F, 58B-34-F, 78B-34-F or 78B-99-F) (Additional file 4: Table S1). These two fragments were ligated by homologous recombination. The resulting vectors pV61, pV92, pV112 and pV190 are pXT1-CGMMV derivatives that include a duplicated copy of 61-bp, 92-bp, 112-bp and 190-bp putative CGMMV CP SGP respectively and a single restriction site $(\mathrm{BamHI})$ between the duplicated CP SGP.

\section{Insertion of different PDS fragments into the CGMMV-based vector}

For a VIGS test with $P D S$ as the target gene, a series of pV1a23, pV92, pV112 and pV190-based vectors harboring different $P D S$ fragments of varied sizes were constructed. Twelve primer sets CuPDS-HindIII-F/R, CuPDS-HindIII-2F/2R, CuPDS-HindIII-3F/3R, 58-150F/R, 78-34-150F/R, 78-150-F/R, 58-213-F/-R, 78-34213F/R, 78-213-F/R, 78-300-F/R, 78-146 N-F/R and 78-215 N-F/R were designed to amplify 114-, 150-, 213-, and 300-bp fragments of the cucurbit PDS gene and 146-, 215-bp fragments of the $N$. benthamiana PDS gene, respectively (Additional file 4: Table S1). These resulting fragments were inserted into digested pV1a23 with HindIII and pV92, pV112, pV190 with BamHI in sense orientation by homologous recombination, respectively. The resulting three $\mathrm{pV} 1 \mathrm{a} 23-$ derived and ten $\mathrm{pV} 92, \mathrm{pV} 112$ and pV190-derived constructs were named pV1a23PDS114, pV1a23-PDS213, pV1a23-PDS300, pV92PDS150, pV92-PDS213, pV112-PDS150, pV112-PDS213, pV190-PDS150, pV190-PDS213, pV190-PDS300, pV190NbPDS146 and pV190-NbPDS215. pV190-PDS69, a construct carrying a 69-bp fragment (dsRNA hairpin structure) of the cucurbit $P D S$ gene, was amplified using pV190 as template and three primers sets 78-69P-X/ CG-4R, CG-4F/78-69P-S and 3R/ TxR R , respectively (Additional file 4: Table S1).

\section{Agroinfiltration and sap inoculation}

All constructs were introduced into Agrobacterium tumefaciens strain GV3101 by freeze-thaw transformation, then single clones were picked up and transferred into $200 \mu \mathrm{L}$ LB liquid media containing kanamycin $\left(50 \mu \mathrm{g} \mathrm{mL}^{-1}\right)$ and rifampicin $\left(50 \mu \mathrm{g} \mathrm{mL}^{-1}\right)$ and cultured overnight in a shaker at $28{ }^{\circ} \mathrm{C}$. The bacterium culture was mixed with $L B$ at a 1:100 ratio and cultured in a shaker overnight, followed by centrifugation at $6000 \mathrm{~g}$ for $5 \mathrm{~min}$ to collect the bacteria. The bacteria were resuspended in inducing buffer solution containing $10 \mathrm{mmol} \mathrm{L}^{-1} \mathrm{MgCl}_{2}$, $10 \mathrm{mmol} \mathrm{L}{ }^{-1} \mathrm{MES}$, and $100 \mu \mathrm{mol} \mathrm{L}^{-1}$ Acetosyringone, and the final $\mathrm{OD}_{600}$ value was adjusted to $0.8-1$. The cells were maintained at room temperature $\left(25^{\circ} \mathrm{C}\right)$ for at least $2 \mathrm{~h}$ before agroinoculation. The upper 2-3 leaves of $N$. benthamiana at the 6-8 leaf stage and cotyledons from 14-day-old cucurbit seedlings were infiltrated with the $A$. tumefaciens suspension using a 1-mL syringe.

In order to verify whether the silencing effect of these vectors could be passaged, the sap from leaves of the agroinfiltrated melon plants displaying obvious photobleaching was used to rub-inoculate cotyledons and the first true leaf (L1) of the melon plants. Each experiment was repeated at least three times, with 9 plants for each construct in each experiment.

\section{DAS-ELISA and RT-PCR}

After agroinfiltration and sap inoculation, CGMMV in inoculated plants was detected by DAS-ELISA and RTPCR at specific time points. DAS-ELISA was performed to detect CGMMV accumulation using an ELISA kit (Adgen, Auchincruive, UK). For RT-PCR, total RNA was extracted from cucurbit (watermelon, melon, cucumber and bottle gourd) and $N$. benthamiana leaf tissues using the RNA simple kit (Tiangen Biotech, Beijing, China) and then first-strand cDNA was synthesized from $1 \mu \mathrm{g}$ total RNA using an oligo dT primer according to the protocol of PrimescriptII RT (TAKARA). PCR was performed with primer set 5574F and 3UTR that flanked the foreign insert to detect CGMMV and asses the stability of the pV190 and foreign inserts of CGMMV-based vectors (Additional file 4: Table S1).

\section{qRT-PCR analysis}

qRT-PCR was performed to measure the mRNA expression level of the endogenous PDS genes using the SYBR Green I Master (Roche) in either N. benthamiana or cucurbit plants inoculated with CGMMV-based vectors at specific time points. The first-strand cDNA was synthesized from $1 \mu \mathrm{g}$ total RNA using an oligo dT primer according to the protocol of PrimeScript ${ }^{\mathrm{TM}} \mathrm{RT}$ reagent Kit with gDNA Eraser (TAKARA). The expression level of $P D S$ of cucumber, melon, gourd and watermelon was determined using primer sets CuPDS-679F/CuPDS$906 \mathrm{R}$ and wate-q-F/R, respectively, designed to prime outside the region targeted for silencing (Additional file 4: Table S1). Expression of the actin gene by primer 
set cumsactin-F/R (Additional file 4: Table S1) was used as an internal control of cucumber, melon, gourd plants. The $C l C A C$ gene was used as an internal control of watermelon plants using primer pairs Cla016178-F/R [47]. The primer set NbPDS-qF/R was designed for detecting the expression of $P D S$ in $N$. benthamiana, and the expression of the GAPDH gene analyzed by the primer set GAPDHqRT-F/R was referred as an internal control (Additional file 4: Table S1). The expression PDS was calculated using the $2^{-\Delta \Delta \mathrm{CT}}$ method [48]. The expression level of $P D S$ in the negative control (pV190) was set to an arbitrary value (1.0) to calculate the relative expression levels of the other samples, with 3 replicates used for each sample.

\section{Accession numbers}

Sequence data from this article can be found in the GenBank or Cucurbit Genomics Database (https://cucurbitge nomics.org/) under the following accession numbers: CGMMV (KC851866); Nicotiana benthamiana PDS (EU165355); Cucumis sativus PDS (XM_011654729); Cucumis melo PDS (NM_001297530); Citrullus lanatu PDS (Cla010898; ClCG07G015130); Lagenaria siceraria PDS (Lsi07G003470).

\section{Supplementary information}

Supplementary information accompanies this paper at https://doi. org/10.1186/s13007-020-0560-3.

Additional file 1: Table S2. The infection analysis of pV1a23 (insertion sites behind the viral CP gene) vector and modified CGMMV-based vector containing a duplicated copy of the 61-, 92-, 112- and 190-bp putative CGMMV CP SGP.

Additional file 2: Fig. S1. The sequence similarities of 114-, 150- , $213-$ and 300-bp PDS gene fragments in four cucurbit species. a, b, c and d correspond to PDS fragments of 114-, 150- , 213- and 300-bp, respectively, in the four cucurbit species. HG, HUG, XG and TG represented cucumber, bottle gourd, watermelon and melon, respectively.

Additional file 3: Fig. S2. Silencing efficiency of different length inserts (PDS) using the pV190 VIGS vector in N. benthamiana. Fragments of $146 \mathrm{bp}, 215 \mathrm{bp}$ were separately cloned into pV190 VIGS vector. (a) The silencing phenotypes were observed at $14 \mathrm{dpi}$. Bar $=1 \mathrm{~cm}$. (b) The relative expression level of PDS mRNA determined by real-time qRT-PCR.

Additional file 4: Table S1. Primers used in this study.

\section{Acknowledgements}

This research was supported by the National Natural Science Foundation of China (31571247), the grants from the earmarked fund for the China Agriculture Research System (CARS-26-13), and the Agricultural Science and Technology Innovation Program (ASTIP), Chinese Academy of Agricultural Sciences (CAAS-ASTIP-2018-ZFRI-08).

\section{Authors' contributions}

QG and ML conceived the original idea and designed the experiments. ML, $Z \mathrm{~L}, \mathrm{LL}$ and $\mathrm{BK}$ performed the experiments and analyzed the data. ML. wrote the manuscript. QG, NH and MA. revised the manuscript. QG agrees to serve as the author responsible for contact and ensures communication. All authors have read and approved the final manuscript.

\section{Availability of data and materials}

All data generated or analyzed during this study are available in this published article.

Ethical approval and consent to participate

Not applicable.

\section{Consent for publication}

All authors agreed to publish this manuscript.

\section{Competing interests}

The authors declare that they have no competing interests.

\section{Author details}

${ }^{1}$ Zhengzhou Fruit Research Institute, Chinese Academy of Agricultural Sciences, Zhengzhou 450009, People's Republic of China. ${ }^{2}$ Huazhong Agricultural University, Wuhan 430070, People's Republic of China. ${ }^{3}$ Centro de Edafologia y Biologia Aplicada del Segura (CEBAS)-CSIC, Apdo. Correos 164, Espinardo, 30100 Murcia, Spain.

Received: 12 November 2019 Accepted: 23 January 2020

Published online: 03 February 2020

\section{References}

1. Renner SS, Schaefer H. Phylogeny and evolution of the Cucurbitaceae. Genetics and genomics of Cucurbitaceae: Springer; 2016. p. 13-23.

2. Grumet R, Garcia-Mas J, Katzir N. Cucurbit genetics and genomics: a look to the future. Genetics and Genomics of Cucurbitaceae. Berlin: Springer; 2017. p. 409-415.

3. Zheng Y, Wu S, Bai Y, Sun H, Jiao C, Guo S, et al. Cucurbit genomics database (CuGenDB): a central portal for comparative and functional genomics of cucurbit crops. Nucleic Acids Res. 2018;47(D1):D1128-D1136136.

4. Guo S, Zhang J, Sun H, Salse J, Lucas WJ, Zhang H, et al. The draft genome of watermelon (Citrullus lanatus) and resequencing of 20 diverse accessions. Nat Genet. 2013:45(1):51.

5. Garcia-Mas J, Benjak A, Sanseverino W, Bourgeois M, Mir G, González VM, et al. The genome of melon (Cucumis melo L.). Proceedings of the National Academy of Sciences. 2012; 109(29): 11872-7.

6. Huang S, Li R, Zhang Z, Li L, Gu X, Fan W, et al. The genome of the cucumber, Cucumis sativus L. Nat Genet. 2009;41(12):1275.

7. Choi PS, Soh WY, Kim YS, Yoo OJ, Liu JR. Genetic transformation and plant regeneration of watermelon using Agrobacterium tumefaciens. Plant Cell Rep. 1994;13(6):344-8.

8. Baulcombe DC. Fast forward genetics based on virus-induced gene silencing. Curr Opin Plant Biol. 1999;2(2):109-13.

9. Ruiz MT, Voinnet O, Baulcombe DC. Initiation and maintenance of virusinduced gene silencing. Plant Cell. 1998;10(6):937-46.

10. Liu Y, Schiff M, Dinesh-Kumar S. Virus-induced gene silencing in tomato. Plant J. 2002;31(6):777-86.

11. Igarashi A, Yamagata K, Sugai T, Takahashi Y, Sugawara E, Tamura A, et al. Apple latent spherical virus vectors for reliable and effective virus-induced gene silencing among a broad range of plants including tobacco, tomato, Arabidopsis thaliana, cucurbits, and legumes. Virology. 2009;386(2):407-16.

12. Ding XS, Schneider WL, Chaluvadi SR, Mian MR, Nelson RS. Characterization of a Brome mosaic virus strain and its use as a vector for gene silencing in monocotyledonous hosts. Mol Plant Microbe Interact. 2006;19(11):1229-39.

13. Zhang C, Bradshaw JD, Whitham SA, Hill JH. The development of an efficient multipurpose bean pod mottle virus viral vector set for foreign gene expression and RNA silencing. Plant Physiol. 2010;153(1):52-655.

14. Sempere RN, Gómez $P$, Truniger $V$, Aranda MA. Development of expression vectors based on pepino mosaic virus. Plant Methods. 2011;7(1):6.

15. Wang R, Yang X, Wang N, Liu X, Nelson RS, Li W, et al. An efficient virusinduced gene silencing vector for maize functional genomics research. Plant J. 2016;86(1):102-15.

16. Liu N, Xie K, Jia Q, Zhao J, Chen T, Li H, et al. Foxtail mosaic virus-induced gene silencing in monocot plants. Plant Physiol. 2016;171(3):1801-7. 
17. Zhao F, Lim S, Igori D, Yoo RH, Kwon S-Y, Moon JS. Development of tobacco ringspot virus-based vectors for foreign gene expression and virus-induced gene silencing in a variety of plants. Virology. 2016:492:166-78.

18. Liao JJ, Wang CH, Xing QJ, Li YY, Liu XF, Qi HY. Overexpression and VIGS system for functional gene validation in oriental melon (Cucumis melo var. makuwa Makino). Plant Cell, Tissue Organ Cult (PCTOC). 2019;137(2):275-84.

19. Bu R, Wang $R$, Wei $Q$, Hu H, Sun $H$, Song $P$, et al. Silencing of glycerol3-phosphate acyltransferase 6 (GPAT6) gene using a newly established virus induced gene silencing (VIGS) system in cucumber alleviates autotoxicity mimicked by cinnamic acid (CA). Plant Soil. 2019;438(1-2):329-46.

20. Dombrovsky A, Tran-Nguyen LT, Jones RA. Cucumber green mottle mosaic virus: rapidly increasing global distribution, etiology, epidemiology, and management. Annu Rev Phytopathol. 2017;55:231-56.

21. Liu L, Peng B, Zhang Z, Wu Y, Miras M, Aranda MA, et al. Exploring different mutations at a single amino acid position of Cucumber green mottle mosaic virus replicase to attain stable symptom attenuation. Phytopathology. 2017;107(9):1080-6.

22. Ugaki M, Tomiyama M, Kakutani T, Hidaka S, Kiguchi T, Nagata R, et al. The complete nucleotide sequence of cucumber green mottle mosaic virus (SH strain) genomic RNA. J Gen Virol. 1991;72(7):1487-95.

23. Ooi A, Tan S, Mohamed R, Rahman NA, Othman RY. The full-length clone of cucumber green mottle mosaic virus and its application as an expression system for hepatitis B surface antigen. J Biotechnol. 2006;121(4):471-81.

24. Teoh P-G, Ooi A-S, AbuBakar S, Othman RY. Virus-specific read-through codon preference affects infectivity of chimeric cucumber green mottle mosaic viruses displaying a dengue virus epitope. BioMed Res Int. 2009; 2009

25. Kumagai MH, Donson J, Della-Cioppa G, Harvey D, Hanley K, Grill L. Cytoplasmic inhibition of carotenoid biosynthesis with virus-derived RNA. Proc Natl Acad Sci. 1995;92(5):1679-83.

26. Jailani AAK, Solanki V, Roy A, Sivasudha T, Mandal B. A CGMMV genomereplicon vector with partial sequences of coat protein gene efficiently expresses GFP in Nicotiana benthamiana. Virus Res. 2017;233:77-85.

27. Zheng H, Xiao C, Han K, Peng J, Lin L, Lu Y, et al. Development of an agroinoculation system for full-length and GFP-tagged cDNA clones of cucumber green mottle mosaic virus. Adv Virol. 2015;160(11):2867-72.

28. Mei L, Liu LM, Wu HJ, Kang BS, Gu QS. Mapping subgenomic promoter of coat protein gene of Cucumber green mottle mosaic virus. J Integr Agric. 2020;19(1):153-63.

29. Mei Y, Zhang C, Kernodle BM, Hill JH, Whitham SA. A Foxtail mosaic virus vector for virus-induced gene silencing in maize. Plant Physiol. 2016;171(2):760-72

30. Avesani L, Marconi G, Morandini F, Albertini E, Bruschetta M, Bortesi L, et al. Stability of Potato virus $X$ expression vectors is related to insert size: implications for replication models and risk assessment. Transgenic Res. 2007;16(5):587-97.

31. Dickmeis C, Fischer R, Commandeur U. Potato virus X-based expression vectors are stabilized for long-term production of proteins and larger inserts. Biotechnol J. 2014;9(11):1369-79.

32. Liu E, Page JE. Optimized cDNA libraries for virus-induced gene silencing (VIGS) using tobacco rattle virus. Plant Methods. 2008;4(1):5.
33. Lacomme C, Hrubikova K, Hein I. Enhancement of virus-induced gene silencing through viral-based production of inverted-repeats. Plant J. 2003;34(4):543-53.

34. Alvarado $\mathrm{VY}$, Scholthof HB. AGO2: a new Argonaute compromising plant virus accumulation. Front Plant Sci. 2012;2:112

35. Molnar A, Melnyk CW, Bassett A, Hardcastle TJ, Dunn R, Baulcombe DC. Small silencing RNAs in plants are mobile and direct epigenetic modification in recipient cells. Science. 2010;328(5980):872-5.

36. Palauqui JC, Elmayan T, Pollien JM, Vaucheret $\mathrm{H}$. Systemic acquired silencing: transgene-specific post-transcriptional silencing is transmitted by grafting from silenced stocks to non-silenced scions. EMBO J. 1997;16(15):4738-45.

37. Dunoyer $P$, Himber $C$, Voinnet O. DICER-LIKE 4 is required for RNA interference and produces the 21-nucleotide small interfering RNA component of the plant cell-to-cell silencing signal. Nat Genet. 2005;37(12):1356.

38. Bouché $N$, Lauressergues $D$, Gasciolli V, Vaucheret $H$. An antagonistic function for Arabidopsis DCL2 in development and a new function for DCL4 in generating viral siRNAs. EMBO J. 2006;25(14):3347-56.

39. Donaire L, Barajas D, Martínez-García B, Martínez-Priego L, Pagán I, Llave C. Structural and genetic requirements for the biogenesis of tobacco rattle virus-derived small interfering RNAs. J Virol. 2008;82(11):5167-77.

40. Zhang Z, Wang J, Zhang R, Huang R. The ethylene response factor AtERF98 enhances tolerance to salt through the transcriptional activation of ascorbic acid synthesis in Arabidopsis. Plant J. 2012;71(2):273-87.

41. Burch-Smith TM, Anderson JC, Martin GB, Dinesh-Kumar SP. Applications and advantages of virus-induced gene silencing for gene function studies in plants. Plant J. 2004;39(5):734-46.

42. Lu R, Malcuit I, Moffett P, Ruiz MT, Peart J, Wu AJ, et al. High throughput virus-induced gene silencing implicates heat shock protein 90 in plant disease resistance. The EMBO J. 2003;22(21):5690-9.

43. Fu DQ, Zhu BZ, Zhu HL, Jiang WB, Luo YB. Virus-induced gene silencing in tomato fruit. Plant J. 2005;43(2):299-308.

44. Ryu CM, Anand A, Kang L, Mysore KS. Agrodrench: a novel and effective agroinoculation method for virus-induced gene silencing in roots and diverse Solanaceous species. Plant J. 2004;40(2):322-31.

45. Senthil-Kumar M, Mysore KS. New dimensions for VIGS in plant functional genomics. Trends Plant Sci. 2011;16(12):656-65.

46. Ratcliff F, Martin-Hernandez AM, Baulcombe DC. Technical advance: tobacco rattle virus as a vector for analysis of gene function by silencing. Plant J. 2001;25(2):237-45

47. Kong Q, Yuan J, Gao L, Zhao L, Cheng F, Huang Y, et al. Evaluation of appropriate reference genes for gene expression normalization during watermelon fruit development. PLoS ONE. 2015;10(6):e0130865.

48. Livak KJ, Schmittgen TD. Analysis of relative gene expression data using real-time quantitative $P C R$ and the $2-\triangle \triangle C T$ method. Methods. $2001 ; 25(4): 402-8$

\section{Publisher's Note}

Springer Nature remains neutral with regard to jurisdictional claims in published maps and institutional affiliations.

Ready to submit your research? Choose BMC and benefit from

- fast, convenient online submission

- thorough peer review by experienced researchers in your field

- rapid publication on acceptance

- support for research data, including large and complex data types

- gold Open Access which fosters wider collaboration and increased citations

- maximum visibility for your research: over 100M website views per year

At $\mathrm{BMC}$, research is always in progress.

Learn more biomedcentral.com/submissions 